\title{
231622
}

UCRL-JC-127298 Pt 1

PREPRINT

\section{Theory of Longitudinal Beam Halo in RF Linacs: \\ I. Core/Test-Particle Formulation}

\author{
J. J. Barnard \\ S. M. Lund
}

This paper was prepared for oubmittal to the

1997 Particle Accelerator Conference

Vancouver, B.C., Canada

May 12-16, 1997

May 9, 1997

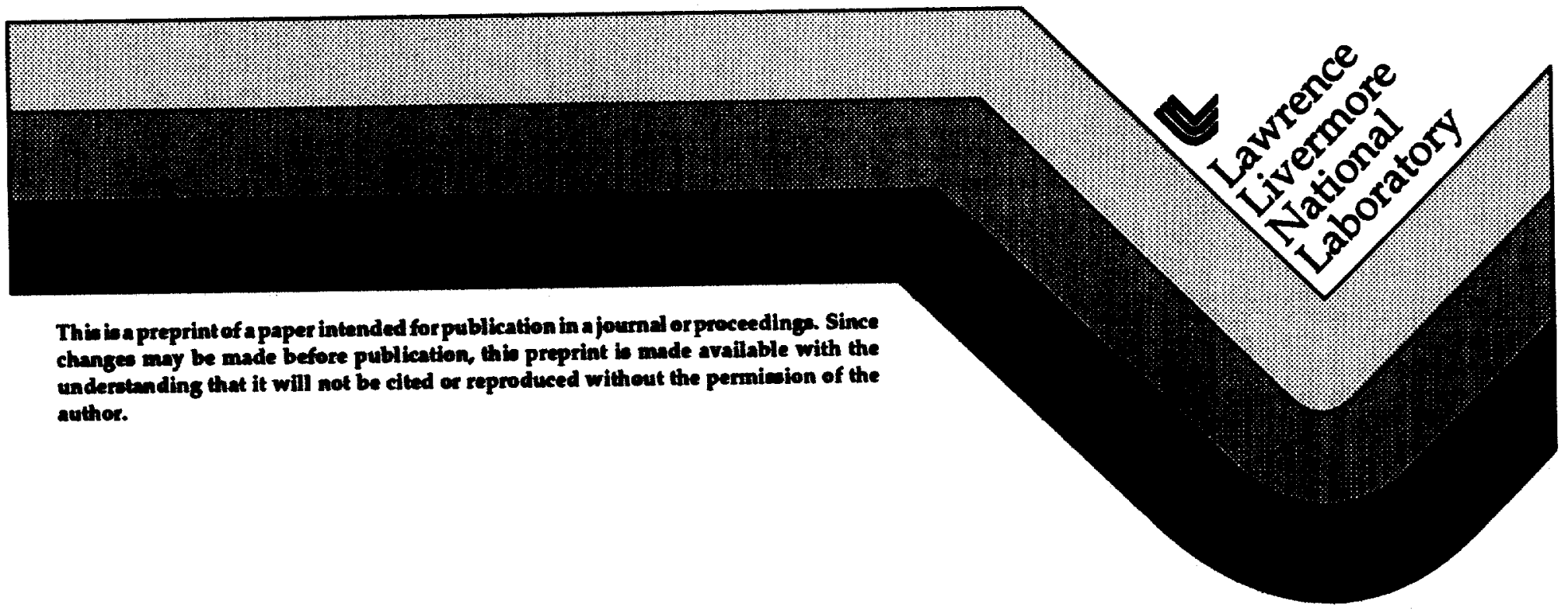




\section{DISCLAIMER}

This document was prepared as an account of work sponsored by an agency of the United States Government. Neither the United States Government nor the University of California nor any of their employees, makes any warranty, express or implied, or assumes any legal liability or responsibility for the accuracy, completeness, or usefulness of any information, apparatus, product, or process disclosed, or represents that its use would not infringe privately owned rights. Reference herein to any specific commercial product, process, or service by trade name, trademark, manufacturer, or otherwise, does not necessarily constitute or imply its endorsement, recommendation, or favoring by the United States Government or the University of California. The views and opinions of authors expressed herein do not necessarily state or reflect those of the United States Government or the University of California, and shall not be used for advertising or product endorsement purposes. 
THEORY OF LONGITUDINAL BEAM HALO IN RF LINACS: I. CORE/TEST-PARTICLE FORMULATION*

John J. Barnard and Steven M. Lund

Lawrence Livermore National Laboratory, L-440, Livermore, CA 94550

Abstract

For intense beams, the analysis of tenuous halo components of the particle distribution that surround the main core of the distribution can be challenging. So-called core/test particle models in which a test particle is evolved in the applied and space-charge forces of the beam core have been instrumental in understanding the structure and extent of transverse beam halo produced by resonant particle interactions with the oscillating space-charge forces of a mismatched beam core. Here we present a core/test particle model developed for the analysis of longitudinal beam halo in intense, ion-beam rf linacs. Equations of motion are derived for a test particle moving interior to, and exterior to, a uniform density ellipsoidal beam bunch. Coupled transverse-longitudinal mismatch modes of the ellipsoidal beam envelope are analyzed. Typical parameters suggest the possibility of a low-order resonant interaction between longitudinal particle oscillations and a low-frequency envelope mode. Properties of this resonance are analyzed in an accompanying paper by the authors in these proceedings ${ }^{1}$.

\section{INTRODUCTION}

Ion linacs with high average current are being considered for applications such as the production of tritium and the transmutation of radioactive wastes. Because beam particle loses can be problematic in these applications due to activation of the accelerator, etc., the structure and control of the halo components of the particle distribution is a critical issue. Recently, the understanding of transverse $(1)$ beam halo has been advanced through analytic theory and numerical simulations ${ }^{2-5}$. In this and the accompanying article ${ }^{1}$, we present theoretical and numerical work on longitudinal (II) beam halo. Longitudinal halo particles are an issue of concern because such particles can have largeamplitude $\|$ oscillations about the synchronous particle, causing a degradation of $\|$ beam quality and possibly particle loss should the oscillation result in a loss of particle synchronisim with the rf fields. Moreover, the control of such halo losses could be challenging since the phase width of a beam bunch in the rf bucket cannot be made arbitrarily small in most intense-beam applications. In contrast, loss of $\perp$ halo particles can be mitigated, though with increased cost, through the use of large-aperture structures.

The core/particle model developed applies to a coasting ellipsoidal beam bunch that is continuously focused and has azimuthal symmetry. The

"Work performed under the auspices of the U.S. D.O.E. by LLNL under contract W-7405-ENG-48 space-charge of the bunch is assumed to remain uniformly distributed while undergoing coupled $\perp$ and II envelope mismatch oscillations, and details of the bunch velocity distribution are left unspecified. Selffield forces associated with the bunch are analytically calculated to obtain coupled equations of motion of a general test particle undergoing both $\perp$ and \| oscillations. These equations are employed to analyze \| halo of on-axis particles (no $\perp$ motion) both with and without nonlinear rf focusing, and to analyze effects of $\perp / \|$ coupling on halo particles (both $\perp$ and $\|$ motion).

To illustrate results, we employ the beam and accelerator parameters summarized in the Table. These parameters represent the $100 \mathrm{MeV}$ and $1.2 \mathrm{GeV}$ points of a conceptual coupled-cavity proton linac (normal conducting design) for the Accelerator Production of Tritium (APT) project ${ }^{5}$. In this design, an intense proton beam is accelerated from $100 \mathrm{MeV}$ to $1.3 \mathrm{GeV}$ over 1060 meters, and beam halo is an issue of concern.

\begin{tabular}{|c|c|c|c|}
\hline Proton Energy, $\mathcal{E}_{0}$ & $\mathrm{GeV}$ & 0.1 & 1.0 \\
\hline Bunch Current, $I$ & $\mathbf{m A}$ & 200 & 200 \\
\hline Sync. Particle Phase, $\phi_{2}$ & degrees & -30 & -30 \\
\hline If Frequency, $\nu$ & $\mathbf{M H z}$ & 700 & 700 \\
\hline Betatron "Freq.", $k_{\beta 0}$ & $\mathrm{rad} / \mathrm{m}$ & 1.04 & 0.204 \\
\hline Synchrotron "Freq.", $k_{80}$ & $\mathrm{rad} / \mathrm{m}$ & 0.30 & 0.041 \\
\hline$\perp$ Norm. Emit., $\gamma_{s} \beta_{s} \epsilon_{s, r m e}$ & $\mathbf{m m}-\mathbf{m r}$ & 0.24 & 0.24 \\
\hline$\|$ Norm. Emit., $\gamma_{g} \beta_{s} \epsilon_{s, r m e}$ & $\mathbf{m m}-\mathbf{m r}$ & 0.58 & 0.62 \\
\hline
\end{tabular}

Table APT parameters in coupled cavity linac.

\section{THEORETICAL MODEL}

We consider an isolated ellipsoidal beam bunch composed of a single species of ion of charge $q$ and mass $m$. The bunch is centered about a synchronous particle with phase $\phi=\phi$, relative to the peak of the synchronous space harmonic of the full if wave. Acceleration is neglected, and the synchronous particle has axial kinetic energy $\mathcal{E}_{s}=$ const. Beam focusing is provided transversely by a constant, linear applied field that represents the average effect of an alternating gradient focusing lattice, and longitudinally by a continuous sinusoidal wave that represents the average effect of the synchronous if space harmonic. The bunch is taken to be azimuthally symmetric $(\partial / \partial \theta=0)$ and to have uniformly distributed charge-density $\bar{\rho}=$ const interior to a sharp ellipsoidal envelope specified by $\left(x_{\perp} / r_{\perp}\right)^{2}+\left(\Delta z / r_{z}\right)^{2}=1$, and zero charge-density exterior to the envelope. Here, $x_{\perp}$ and $\Delta z$ are the $\perp$ and $\|$ coordinates relative to the synchronous particle, and $r_{\perp}$ and $r_{z}$ are the $\perp$ and $\|$ radii of the ellipsoidal beam envelope. The $\|$ coordinate $\Delta z$ is related to the If phase $\phi$ by $\Delta z=-(\beta, \lambda / 2 \pi) \Delta \phi$, where $\Delta \phi \equiv \phi-\phi s$, $\lambda$ is the vacuum wavelength of the rf wave $(\lambda=c / \nu$, 
where $c$ is the speed of light in vacuo and $\nu$ is the rf frequency), and $\beta_{s}$ and $\gamma_{s}=1 / \sqrt{1-\beta_{s}^{2}}$ denote the usual synchronous particle relativistic factors. Denoting the time average current of the bunch over an if period by $I$, the charge-density in the bunch is $\bar{\rho}=3 I \lambda / 4 \pi r_{\perp}^{2} r_{z} c$.

The $\perp$ and $\|$ forces acting on a particle due to electrostatic and leading-order self-magnetic fields can be calculated in the absence of material boundaries as ${ }^{6}$

$$
\begin{aligned}
F_{\perp} & =\frac{q \tilde{\rho}}{2 \epsilon_{0} \gamma^{2}}\left[\frac{\alpha^{2}}{\left(\alpha^{2}+\chi\right)^{2}(1+\chi)^{1 / 2}}-F(\chi, \alpha)\right] \mathrm{x}_{\perp}, \\
\Delta F_{z} & =\frac{q \tilde{P}}{\epsilon_{0}} F(\chi, \alpha) \Delta z .
\end{aligned}
$$

Here, $\epsilon_{0}$ is the permittivity of free-space,

$$
\begin{aligned}
& F(\chi, \alpha) \equiv \\
& \begin{cases}\frac{\alpha^{2}}{1-\alpha^{2}}\left[\frac{1}{\sqrt{1-\alpha^{2}}} \tanh ^{-1}\left(\frac{\sqrt{1-\alpha^{2}}}{\sqrt{1+\chi}}\right)-\frac{1}{\sqrt{1+\chi}}\right], & \alpha<1, \\
\frac{1}{3(1+\chi)^{3 / 2},} & \alpha=1, \\
\frac{\alpha^{2}}{\alpha^{2}-1}\left[\frac{1}{\sqrt{1+\chi}}-\frac{1}{\sqrt{\alpha^{2}-1}} \tan ^{-1}\left(\frac{\sqrt{\alpha^{2}-1}}{\sqrt{1+\chi}}\right)\right], & \alpha>1,\end{cases}
\end{aligned}
$$

$\alpha=r_{1} / \gamma_{a} r_{2}$ is the aspect ratio of the ellipsoidal beam as measured in the synchronous-particle frame, and $\chi$ is the positive root of the equation

$$
\frac{\alpha^{2}\left(x_{1} / r_{3}\right)^{2}}{\alpha^{2}+x}+\frac{\left(\Delta z / r_{s}\right)^{2}}{1+\chi}=1
$$

for a particle exterior to the bunch envelope [i.e., $\left.\left(x_{1} / r_{z}\right)^{2}+\left(\Delta z / r_{z}\right)^{2}>1\right]$, and $\chi=0$ for a particle interior to the bunch [i.e., $\left.\left(x_{\perp} / r_{z}\right)^{2}+\left(\Delta z / r_{x}\right)^{2}<1\right]$. For the special cases of a particle in the beam, $x=0$, and the self-field forces (1) reduce to the familiar linear expressions ${ }^{7,8}$, and for a spherical beam bunch in the beam frame $\left(r_{\perp}=\gamma_{2} r_{z}\right)$, the forces fall off quadratically with the required inverse-square form.

Within the paraxial approximation, the $\perp$ and $\|$ equations of motion of a single test particle moving in the applied focusing fields and self-field defocusing forces (1) of the bunch can be expressed as

$$
\begin{gathered}
\frac{d}{d s} x_{\perp}=x_{\perp}^{\prime}, \quad \frac{d}{d s} \Delta \phi=-\frac{2 \pi}{\gamma_{!}^{3} \beta 3} \frac{\Delta \varepsilon}{m c^{2}} \\
\frac{d}{d s} x_{\perp}^{\prime}=-k_{\beta 0}^{2} x_{\perp}+\frac{K_{30}}{2 r_{\perp}^{2} r_{s}}\left[\frac{\alpha^{2}}{\left(\alpha^{2}+\chi\right)(1+x)^{1 / 2}}-F(x, \alpha)\right] x_{\perp} \\
\frac{d}{d s}\left(\frac{\Delta \varepsilon}{m c^{2}}\right)=\frac{q E_{0}}{m c^{2}}\left[\cos \left(\Delta \phi+\phi_{s}\right)-\cos \phi_{s}\right] \\
-\frac{\beta_{3}^{3} \gamma_{s}^{3}}{2 \pi} \frac{\lambda K_{3 D}}{r_{\perp}^{2} r_{z}} F(\chi, \alpha) \Delta \phi .
\end{gathered}
$$

Here, $s$ is the axial distance the beam has propagated, $x_{\perp}$ and $x_{\perp}^{\prime}$ are the $\perp$ coordinate and convergence angle of the particle, $\Delta \phi$ and $\Delta \mathcal{E}=\mathcal{E}-\mathcal{E}$, are the $\|$ particle phase and kinetic energy relative to the synchronous particle, $k_{\beta 0}=$ const is the undepressed ( $I=$ $0)$ betatron wavenumber of $\perp$ particle oscillations, $E_{0}$ $\left(E_{0} \rightarrow E_{0} T\right.$ for a standing-wave structure, with $T$ the transit-time factor) is the peak, on-axis $\left(x_{\perp}=0\right)$ field value of rf wave, and $K_{3 D} \equiv 3 q I \lambda / 4 \pi \epsilon_{0} \gamma_{s}^{3} \beta_{s}^{2} m c^{3}$ is a three-dimensional space-charge parameter of dimension length. In general, note that the $\perp$ and $\|$ Eqs. (4) are coupled due to the coordinate dependence in $\chi$. However, for a particle moving within the bunch (i.e., $\chi=0$ ) or along the axes of symmetry of the bunch (i.e., $x_{\perp}=0$ or $\Delta \phi=0=\Delta z$ ), the equations are uncoupled. Finally, for small $\|$ particle excursions from the synchronous particle with $2 \pi|\Delta z| / \beta_{3} \lambda \ll 1$, the axial $\mathrm{rf}$ focusing force becomes linear, i.e.,

$$
\frac{q E_{0}}{\beta_{s}^{2} \gamma_{s}^{3} m c^{2}}\left[\cos \left(\Delta \phi+\phi_{s}\right)-\cos \phi_{s}\right] \longrightarrow k_{s 0}^{2} \Delta z,
$$

where $k_{s 0} \equiv \sqrt{2 \pi q E_{0} \sin \left(-\phi_{s}\right) / \gamma_{s}^{3} \beta_{s}^{3} \lambda m c^{2}}$ is the wavenumber of undepressed "synchrotron" oscillations about the synchronous particle. In the presence of space-charge $(I \neq 0)$ and within the bunch, it is apparent from Eqs. (4) that the characteristic $\perp$ and II spatial frequencies $k_{\beta 0}$ and $k_{s 0}$ are depressed to the values

$$
\begin{aligned}
& k_{\beta}^{2}=k_{\beta 0}^{2}-\frac{K_{s 0}}{2 r_{1}^{2} r_{s}}[1-f(\alpha)], \\
& k_{s}^{2}=k_{s 0}^{2}-\frac{K_{s 0}^{2}}{r_{1}^{2} r_{s}} f(\alpha),
\end{aligned}
$$

where $f(\alpha) \equiv F(\chi=0, \alpha)$ is a beam aspect ratio form factor [corresponding to $f$ in Ref. 7].

\section{ENVELOPE EQUATIONS}

To consistently determine the ellipsoidal bunch radii $r_{\perp}$ and $r_{z}$ in terms of beam and accelerator $p a-$ rameters, it is necessary to derive so-called "envelope equations" for the evolution of $r_{\perp}$ and $r_{x}$. For a uniform density distribution, $r_{1}^{2}=(5 / 2)\left\langle r^{2}\right\rangle$ and $r_{3}^{2}=$ $5\left\langle\Delta z^{2}\right\rangle$, where $\rangle$ indicates an average over the entire 3D distribution function. By taking derivatives of these relations and using the equations of motion (4), the envelope equations

$$
\begin{aligned}
& \frac{e^{2} r_{1}}{d \sigma^{2}}=-k_{\rho 0}^{2} r_{\perp}+\frac{K_{3 D}[1-f(\alpha)]}{2 r_{\perp} r_{3}}+\frac{\epsilon_{f}^{2}}{r_{1}^{3}} \\
& \frac{d^{2} r_{r}}{d r^{2}}=-f_{r f}(\zeta) k_{20}^{2} r_{x}+\frac{K_{30} f(\alpha)}{r_{\perp}^{2}}+\frac{c_{z}^{2}}{r_{s}^{2}}
\end{aligned}
$$

are obtained ${ }^{7,8}$. Here, $f(\alpha)=F(\chi=0, \alpha), \epsilon_{x}^{2} \equiv$ $25\left[\left\langle x^{2}\right\rangle\left(x^{\prime 2}\right\rangle-\left\langle x x^{\prime}\right\rangle^{2}\right]=25 \epsilon_{x, \text { rms }}^{2}$ and $\epsilon_{3}^{2} \equiv$ $25\left[\left\langle\Delta z^{2}\right\rangle\left\langle\Delta x^{\prime 2}\right\rangle-\left\langle\Delta z \Delta z^{\prime}\right\rangle^{2}\right]=25 \epsilon_{x, r m s}^{2}$ are the squares of the unnormalized $\perp$ and $\|$ 3D bunch emittances, and

$$
f_{r f}(\zeta)=\frac{15}{\zeta^{\zeta}}\left[\left(3-\zeta^{2}\right) \sin \zeta-3 \zeta \cos \zeta\right]
$$

with $\zeta \equiv\left(2 \pi / \beta_{s} \lambda\right) r_{x}$ is a nonlinear $\mathrm{rf}$ focusing factor.

Note that Eqs. (7) differs from those in Refs. 7 and 8 by the inclusion of a multiplicative factor $f_{r f}(\zeta)$, which arises when the the external. $\mathrm{rf}$ force is included in an average over the $\|$ coordinate. This factor approaches unity as $\zeta \rightarrow 0$, corresponding to small $\|$ beam extent $r_{z}$ relative to the $\mathrm{rf}$ wavelength $\lambda$. Note that Eqs. (7) are not self-consistent in that the distribution function is assumed to evolve such that its density remains uniform within an ellipsoid, whereas, in general such evolution does not occur. It is hoped that, since the extent of the distribution is not constrained (though the form of the distribution is), the evolution of these envelopes is close to the actual evolution of the rms radii, thereby providing halo particles with approximately correct space-charge impulses. 


\section{LINEAR ENVELOPE MODES}

Denote stationary $(d / d s=0)$ equilibrium (subscript 0 ) solutions to the envelope equations ( 7 ) by $r_{\perp 0}$ and $r_{z 0}$, and assume coupled, sinusoidally varying perturbations about the equilibrium radii of the form $r_{\perp}=r_{\perp 0}+\delta r_{\perp} \mathrm{e}^{i k s}$ and $r_{z}=r_{z 0}+\delta r_{x} \mathrm{e}^{i k s}$ with constant amplitudes $\delta r_{\perp}$ and $\delta r_{z}$. For $\left|\delta r_{\perp, z}\right| / r_{\perp 0,80} \ll 1$ these perturbations can be expanded to leading order in Eq. (7) to obtain the dispersion relation

$$
k^{4}-\left(K_{11}+K_{22}\right) k^{2}+K_{11} K_{22}-K_{12} K_{21}=0
$$

for the mode wavenumber or spatial frequency $k$. Here,

$$
\begin{aligned}
K_{11}= & 4 k_{\rho 0}^{2}-\frac{K_{3 D}}{r_{10}^{2}}\left[1-f\left(\alpha_{00}\right)-\frac{\alpha_{0}}{2} \frac{d f\left(\alpha_{0}\right)}{d \alpha_{0}}\right], \\
K_{22}= & 4 k_{s 0}^{2}\left[f_{r f}\left(\zeta_{0}\right)+\frac{\zeta_{0}}{4} \frac{d f_{r f}\left(\zeta_{0}\right)}{d \zeta_{0}}\right] \\
& -\frac{3 K_{30}}{r_{10}^{2} r_{30}}\left[f\left(\alpha_{0}\right)-\frac{\alpha_{0}}{3} \frac{d f\left(\alpha_{0}\right)}{d \alpha_{0}}\right], \\
K_{12}= & \frac{K_{30}}{2 r_{10}^{2} r_{30}}\left[1-f\left(\alpha_{0}\right)-\alpha_{0} \frac{d f\left(\alpha_{0}\right)}{d \alpha_{0}}\right], \\
K_{21}= & \frac{K_{30}}{r_{10}^{2} r_{s 0}}\left[2 f\left(\alpha_{0}\right)-\alpha_{0} \frac{d f\left(\alpha_{0}\right)}{d \alpha_{0}}\right],
\end{aligned}
$$

are equilibrium parameters. Since Eq. (9) is quadratic in $\boldsymbol{k}^{2}$, two modes are present, a high frequency (HFM, $k \equiv k_{H}$ ) and low frequency mode (LFM, $k \equiv k_{L}$ ), each with a relative amplitude of $\perp$ to $\|$ oscillation given by

$$
R \equiv \frac{\delta r_{1} / r_{10}}{\delta r_{3} / r_{80}}=\frac{K_{12}}{K^{2}-K_{11}} \text {. }
$$

In the Figure, equilibrium and mode properties are presented as a function of bunch current $I$ assuming fixed $\perp$ and $\|$ beam emittances $\epsilon_{x}$ and $\epsilon_{z}$. Parameters correspond to the $100 \mathrm{MeV}$ linac presented in the Table with $I$ varied about the nominal $200 \mathrm{~mA}$ value.

\section{v. CONCLUSIONS}

From Figure a and $c$, it is apparent that for beams in which the $\perp / \|$ applied focusing and equilibrium radii ratios $k_{\beta 0} / k_{10}$ and $r_{x 0} / r_{\perp 0}$ are of order a few, the frequencies of the HFM $\left(k_{H}\right)$ and LFM $\left(k_{L}\right)$ are well separated. (For the more recent superconducting design of APT, the ratio $r_{z 0} / r_{\perp 0}$ is closer to unity ${ }^{9}$ implying less separation of the oscillation frequencies, however). From Figure $c$, note that the LFM is antisymmetric, with $\perp$ envelope excursions much smaller than \| excursions, and 180 degrees out of phase. In contrast, the HFM is symmetric, with \| excursions much smaller than $\perp$ excursions and in-phase. The frequency of the LFM falls between twice the \| particle frequency within the beam $\left(\sim 2 k_{s}\right)$, and twice the undepressed frequency $\left(\sim 2 k_{s 0}\right.$, i.e. twice the $\|$ particle frequency at large $|\Delta z|$ in the linear approximation to the rf force). Particles outside the beam envelope longitudinally will have an oscillation frequency in between $k$, and $k, 0$, suggesting a possible low-order (2:1) resonant interaction between \| particle and envelope motion. This situation is analogous to the transverse case $^{2-4}$ and has analogous implications for longitudinal beam halo. This longitudinal resonance is analyzed in an accompanying paper ${ }^{1}$.
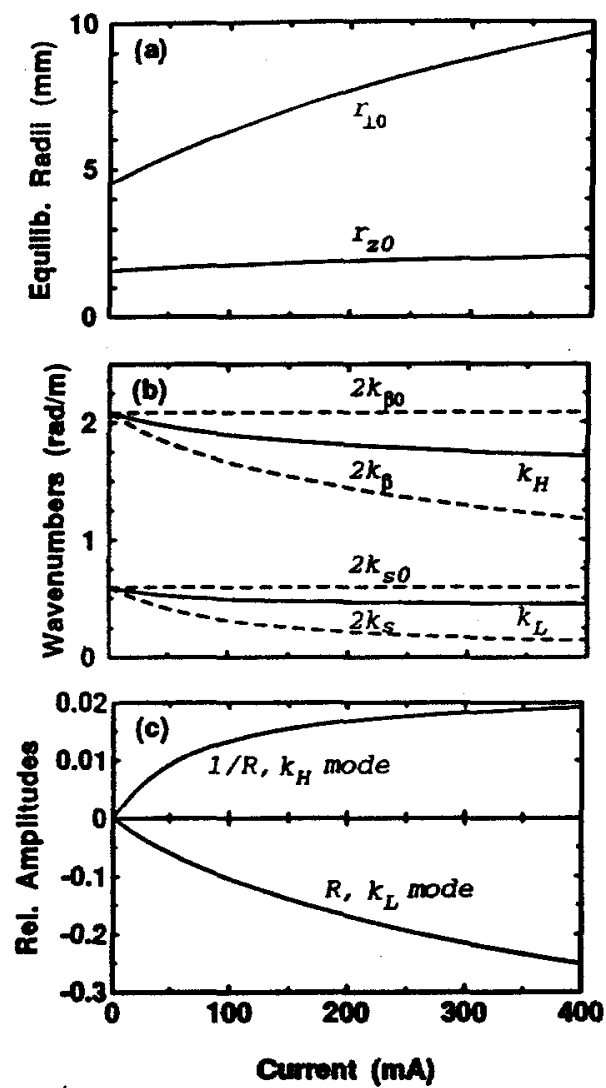

Figure Ellipsoidal beam equilibrium and mode properties as a function of current. a. Equilibrium beam radii $r_{\perp 0}$ and $r_{30}$. b. Wavenumbers for high $\left(k_{H}\right)$ and low $\left(k_{L}\right)$ frequency envelope modes (solid curves), and two times the undepressed and depressed frequencies of $\perp\left(k_{p 0}, k_{p}\right)$ and $\|\left(k_{20}, k_{\rho}\right)$ particle oscillations (dashed curves). c. Ratio $R$ of $\perp$ to $\|$ mode amplitude for the high and low frequency envelope modes.

\section{ACKNOWLEDGMENTS}

The authors wish to thank Rob Ryne, Tom Wangler, and Bob Gluckstern for many valuable discussions on beam halo and their support of this project.

\section{REFERENCES}

[1] S.M. Lund and J.J. Barnard, "Theory of Longitudinal Beam Halo in RF Linacs: II. Envelope/Particle Resonances," these proceedings.

[2] a) J.S. O'Connell, T.P. Wangler, and K.R. Krandall, p. 3657, and b) R.A. Jameson, p. 3926, both in Proc. 1993 Part. Accel. Conf., Washington DC (1993).

[3] R.L. Gluckstern, Phys. Rev. Letters, 73, 1247, (1994).

[4] R.D. Ryne and T.P. Wangler, Proc. of the Accel. Driven Transmutation Tech. Conf., Las Vegas, NV, July 1994.

[5] J Stovall et al., APT Design Review Presentation, Los Alamos National Laboratory, Oct., 1996.

[6] J. J. Barnard and S. M. Lund, "Theory of Longitudinal Beam Halo in High Current Ion RF Linacs," in prep.

[7] T.P. Wangler, "Introduction to Linear Accelerators,"

Los Alamos Report, LA-UR-94-125 (1994).

[8] M. Reiser, "Theory and Design of Charged Particle Beams," (Wiley \& Sons, New York 1994).

[9] T.P. Wangler, private communication (1997). 


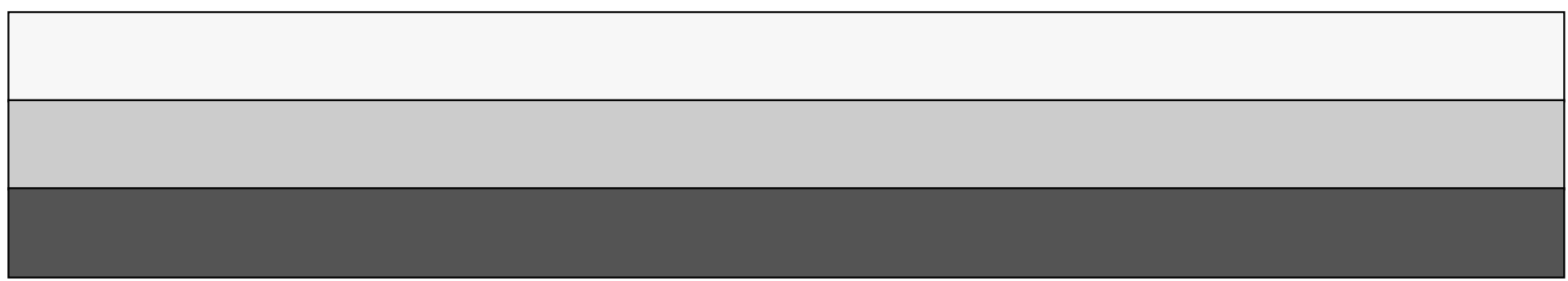

